\title{
GERENCIAMENTO SOCIAL DE RISCOS
}

\author{
SOCIAL MANAGEMENT OF RISKS
}

Adelaide Cássia Nardocci ${ }^{* *}$

\section{RESUMO}

O objetivo é dar subsídios para a melhor compreensão do conhecimento técnico-científico que acompanha as discussões dos parâmetros e dos processos de tomada de decisão nas questões de risco. Realizou-se uma investigação teórica multidisciplinar, fundamentada na literatura disponível sobre 0 assunto, tomando como referências básicas as ferramentas de gerenciamento atualmente empregadas. $O$ material de estudo compõem-se principalmente de reflexões realizadas por diversos autores que abordam a questão. Conclusões: (i) A definição dos critérios fundamentais para as políticas de risco deve incluir uma abordagem ética e procedimentos democráticos, e não apenas considerações técnico-científicas. (ii) O gerenciamento de risco inclui todas as decisões e escolhas sociais, políticas e culturais que se relacionam direta e indiretamente com as questões de risco na nossa sociedade. (iii) É necessário ampliar o debate, no Brasil, não apenas dos critérios e ferramentas, como também das suas implicações sociais, políticas e éticas envolvidas na tomada de decisão sobre aceitabilidade de riscos.

\section{Palavras-chave}

Avaliação de riscos. Gerenciamento de riscos. Políticas sociais de risco.

\section{ABSTRACT}

Given subsidies for a better comprehension on technical and scientific knowledge which follows the discussions about risk as environmental management instrument. This research was characterized of a multidisciplinary theoretical reflection based on the available literature on the subject, ha-

(*) Professora Doutora do Departamento de Saúde Ambiental da Faculdade e Saúde Pública da USP. 
ven as basic references the management tools employed nowadays. The study contents is composed mainly of reflection by authors who study this field. Conclusions: (i) The definition of the fundamental criteria for the risk policies must include an ethical approach and democratic procedures, and not only technical and scientific considerations. (ii) The risk management include all the decisions and social, political and cultural choices which are direct or indirectly related to the risk questions in our society.(iii) Its necessary to improve the debats of risk management tools as its the social, ethics and political implications.

\section{Keywords}

Risk assessment. Risk management. Risk social policy.

\section{INTRODUÇÃO}

O impacto potencial do desenvolvimento tecnológico e das mudanças no estilo de vida bem como o aumento da sensibilidade para os perigos à saúde e segurança têm colocado os riscos e a qualidade ambiental entre uma das maiores preocupações da sociedade atual.

Segundo o sociólogo alemão Beck(1), houve uma mudança gradual no conflito social predominante neste século. $O$ conflito primário, no início do século $X X$, era centrado na distribuição do bem-estar entre os grupos sociais; depois da Segunda Guerra Mundial e particularmente a partir de 1960, o foco mudou para a distribuição de poder na política e na economia. Nos últimos anos, o maior conflito é sobre a distribuição e tolerabilidade dos riscos para os diferentes grupos sociais, regiões e gerações futuras.

O conceito de risco tem-se tornado fundamental, portanto, e apresenta-se como critério central nas negociações, relações e organização da sociedade, quer no âmbito local, nacional ou mesmo global.

No entanto, o processo de tomada de decisões e definição de critérios por uma sociedade ou organizações nas questões de risco é muito complexo e envolve, além da sua própria definição - uma vez que não existe um conceito único e aplicável a todo contexto - a limitação das metodologias científicas de avaliação em considerar dados subjetivos e incertezas, a aceitabilidade social, a distribuição dos riscos e benefícios, a legitimação social dos métodos científicos de avaliação e gerenciamento, bem como a voluntariedade dos riscos.

Essas questões envolvem aspectos éticos relevantes não apenas em relação às responsabilidades sociais e éticas de cientistas em geral e 
especialistas em riscos em particular, como também a ética das relações sociais como um todo(2).

No Brasil, o debate atual sobre essas questões é muito limitado. Metodologias de avaliação, critérios e ferramentas de gerenciamento têm sido trazidos de outros países, sem uma análise da sua aplicabilidade à realidade brasileira e ou das suas implicações sociais e políticas. Dessa forma, o ponto fundamental deste trabalho foi avaliar as várias dimensões dos parâmetros e processos de tomada de decisão envolvidos nas questões de risco.

\section{METODOLOGIA}

Esta investigação caracterizou-se essencialmente por uma reflexão teórica fundamentada na literatura disponivel sobre o assunto, tomando-se como vertente principal de análise as ferramentas de gerenciamento atualmente empregadas. O material de estudo compõe-se principalmente de reflexões realizadas por diversos autores que abordam a questão.

\section{O QUE É GERENCIAMENTO DE RISCOS?}

Existem muitos conceitos e diferentes definições de risco em uso nas mais diversas áreas da ciência atual, como psicologia, economia, ciências médicas, ciências atuariais, engenharia e estatística(3).

De forma resumida, podemos dizer que em face das abordagens existentes sobre riscos, os riscos se dividem em objetivos e subjetivos. Os riscos objetivos são aqueles estimados a partir de cálculos estatísticos e metodologias quantitativas, enquanto os riscos subjetivos são aqueles avaliados com base em julgamentos intuitivos.

Não há consenso entre os especialistas sobre o que compreende exatamente o gerenciamento de riscos. Os especialistas de áreas técnicas, em geral, consideram que a avaliação objetiva de risco é um processo essencialmente técnico e que o gerenciamento de riscos se inicia com o julgamento sobre a aceitabilidade dos níveis de riscos calculados. Mais especificamente, a avaliação é a etapa do entendimento do problema e o gerenciamento a etapa das ações(4). Por outro lado, os trabalhos na área social se referem ao gerenciamento como uma área ampla que abrange todas as atividades técnicas e legais, escolhas e decisões, individuais e sociais, ligadas direta ou indiretamente ao risco.

Uma das principais preocupações em se separar avaliação e gerenciamento de riscos é para distinguir o que é técnico e científico do que é político e social. Será que é possível essa separação? 
O desenvolvimento científico é um processo histórico. A ciência não se desenvolve independentemente do contexto sociopolítico vigente. A definição de prioridades de pesquisa e de montante dos investimentos a serem feitos relaciona-se, em parte, com a demanda social existente e orienta, define e influencia diretamente o desenvolvimento científico. Da mesma forma, o conhecimento científico indica novos caminhos e cria novos valores sociais e políticos. Portanto, há uma relação reflexiva e constante entre a ciência e a sociedade(5).

Além disso, as avaliações de risco carregam incertezas importantes e necessitam de julgamentos e escolhas por parte dos especialistas; tratase de julgamentos essencialmente políticos.

Os estudos da amplificação social do risco mostram que há efetivamente uma associação entre o contexto social e político e os riscos calculados. No caso dos riscos tecnológicos, por exemplo, o número de fatalidades em acidentes industriais em países pobres é superior ao encontrado em paises industrializados(6).

Essa diferença é mostrada pela própria definição objetiva do risco tecnológico e não é apenas subjetiva. Assim, considerar a avaliação de risco como a etapa de entendimento do problema e o gerenciamento como a etapa das ações é uma simplificação, pois um amplo entendimento das questões de risco, necessariamente, inclui uma abordagem do contexto social, político e cultural, dos valores e interesses predominantes de cada ator social envolvido. Isso certamente inviabiliza a definição de fronteiras claras entre o que é estritamente político e social e o que é científico ou técnico.

Essa pretensão de se separar o que é técnico e científico do que é político e social, certamente, pode ser muito mais fruto do pragmatismo técnico e da acentuada crença na racionalidade científica, que caracteriza a sociedade atual, na qual não são admitidas outras formas racionais de resolução de problemas(7).

Além disso, decidir simplesmente sobre se o risco imposto é aceitável induz a uma comparação entre diferentes tipos de risco e não uma entre diferentes alternativas. Qual o objetivo da atividade proposta? Quem a está propondo? Qual a sua importância e em que contexto social e político será inserida? Quais as outras alternativas de que a sociedade dispõe? 0 que exatamente será perdido se esta atividade for rejeitada? Esta discussão precede à questão da aceitabilidade do risco, pois, se a sociedade decidir que não precisa daquela atividade, não será necessário calcular os seus riscos.

Por outro lado, se a sociedade julgar que uma atividade é necessária e que seus riscos devem ser estimados, qual será o papel da avaliação 
técnica nesse processo e que uso será feito dos seus resultados? As respostas a estas perguntas devem também preceder à avaliação, pois as regras devem ser definidas antes do início do jogo e a definição dessas regras e dos papéis e responsabilidades de cada ator social envolvido já fazem parte também do processo de gerenciamento dos riscos.

Portanto, o gerenciamento dos riscos deve ser considerado não apenas como o conjunto de regras e procedimentos que deve ser seguido após a avaliação, com o objetivo de controlar os riscos estimados, mas também como todas as atividades técnicas e legais, bem como o conjunto de todas as decisões e escolhas sociais, políticas e culturais que se relacionam direta ou indiretamente com as questões de risco na nossa sociedade.

Não se pretende, com isso, dizer que as avaliações objetivas de risco têm papel menor neste processo, mas apenas que a busca por decisões não apenas socialmente mais aceitáveis como também mais eficazes do ponto de vista ambiental necessita de uma abordagem mais ampla, e não focada apenas nas discussões de resultados de avaliações. A avaliação do risco representa, apesar das incertezas que carrega, o melhor conhecimento técnico e científico de que se dispõe no momento acerca da questão em estudo, o que não significa que o seu resultado possa ser tomado, de imediato, como absoluto e determinante das decisões.

\subsection{Como Gerenciar Riscos?}

Se o conteúdo do gerenciamento é ampliado, as dificuldades com a definição de critérios e ferramentas normativas e legais que orientem as ações e decisões também o serão.

Há vários parâmetros que são comuns nos processos de tomada de decisão, como risco, benefício, custo e eficácia. O processo de tomada de decisão nas situações de risco sempre esteve relacionado, de certa forma, com o balanço risco-benefício, ou seja, para todo risco há sempre um benefício compensatório. Além disso, a tomada de decisão sobre as medidas de controle a serem implantadas é balanceada em relação ao custo e eficácia, ou seja, a relação entre o custo da medida e a sua eficácia em termos de redução de riscos.

Partindo-se do princípio de que o balanço risco-benefício é parâmetro importante para uma tomada de decisão eficaz, várias questões devem ser colocadas inicialmente. Entre elas: $O$ que são benefícios? A todo risco necessariamente corresponde um benefício, ou existe um nível acima do qual não há benefícios aceitáveis? Como distribuir os riscos e os benefícios?

Benefícios podem ser entendidos como "vantagens, proveitos ou ganhos"(8) que se obtêm a partir de uma atividade. Quando escolhemos uma 
nova tecnologia ou quando um novo produto químico é introduzido no mercado, supõe-se sempre que isso trará novos benefícios à sociedade e a questão central é como estimá-los e como saber se estes compensam os riscos impostos. Da mesma forma que os riscos, os tipos de benefícios também são variados e distintos e podem incluir lucros financeiros, melhoria da qualidade de vida, menores impactos ambientais, empregos etc., e podem ser individuais e/ou sociais, imediatos e de longo prazo.

As dificuldades são como quantificar parâmetros tão distintos, saber se um benefício imediato compensa um risco de longo prazo, ou se um risco imediato poderá ser compensado com benefícios de longo prazo. Benefícios sociais compensam riscos individuais e vice-versa? Além da dificuldade técnica, objetiva das respectivas avaliações, estas questões envolvem aspectos éticos relevantes.

Também como os riscos, os benefícios são construções sociais e também são percebidos diferentemente pelos indivíduos. Muitas vezes, o que é benefício para um grupo pode não sê-lo para outro, e o que hoje é visto como uma vantagem pode não o ser futuramente e vice-versa.

Em termos técnicos, a estimativa dos benefícios de uma atividade é realizada em base econômica(9). Nesta abordagem, a análise de risco é vista como parte de um processo mais amplo de consideração de custobenefício, no qual os riscos são as perdas de utilidade esperadas como resultantes de um evento ou atividade. $O$ objetivo principal é alocar recursos de tal forma a maximizar a sua utilidade para a sociedade(10).

Entre as várias críticas a estas abordagens, como, por exemplo, a que questiona a subjetividade dos julgamentos, duas são mais relevantes. A primeira é que essas abordagens se baseiam em preferências individuais enquanto a maioria das decisões de risco é coletiva. Como avaliar o bemestar social a partir das preferências individuais? A segunda diz respeito às questões éticas que envolvem. Do ponto de vista utilitarista, a maior utilidade, ou seja, o maior aumento proporcional de receita decorrente de uma atividade é encontrada, em geral, entre as comunidades mais pobres, uma vez que, como têm poucos recursos, qualquer acréscimo já se torna significativo. Isso implica que estas comunidades acabam, também, recebendo os niveis mais altos de risco(11).

Mas, por outro lado, os deontologistas defendem que a imposição do risco seria justificada dentro de um contexto de consenso individual, compensações e da eqüidade da distribuição do risco sobre toda a população(11).

Compatibilizar a distribuição dos riscos e benefícios é uma das questões mais complexas para uma política de gerenciamento social de riscos. 
$\mathrm{Na}$ organização atual da nossa sociedade, os riscos e custos são quase sempre sociabilizados enquanto os maiores benefícios são privados. As pessoas que estão expostas aos níveis mais altos de risco, em geral, não são as que recebem os maiores benefícios.

Mas, de qualquer forma, há uma associação entre os riscos e os benefícios percebidos pela população. Ou seja, as pessoas tendem a perceber riscos menores se elas percebem os benefícios maiores. Será que essa associação é diretamente proporcional, ou seja, a população estará sempre disposta a trocar um risco por um benefício ou existem riscos que não poderão ser compensados?

Com aumento da preocupação em relação às questões ambientais, em muitas situações a população tem rejeitado os riscos por não considerar seus benefícios compensatórios. Um exemplo é a rejeição popular ao consumo de alimentos transgênicos, especialmente em alguns países europeus, e ao uso da energia nuclear.

Embora seja precoce ainda fazer generalizações, há uma tendência de a população não aceitar quaisquer riscos e benefícios. Ou seja, em alguns casos, não há limites aceitáveis para os riscos e não há benefícios que os compensem.

Eticamente também é controvertida a troca de benefícios econômicos por perdas de vidas humanas e a definição do valor da vida humana. Decidir quantas mortes podem ser toleradas, em decorrência de um evento qualquer, e quanto vale cada uma dessas vidas é um julgamento de valor muito complexo. O valor da vida humana é calculado, em geral, considerando-se o salário médio das vítimas nos seus últimos anos e a expectativa de vida para esses indivíduos. Esse cálculo, portanto, refere-se basicamente ao quanto deixaram de produzir morrendo antes da hora.

Essa consideração evidencia que a perda de uma vida está essencialmente relacionada a uma perda econômica, cujo valor é determinado em função da classe social, da raça, da ocupação, do sexo etc. Por outro lado, se é considerado que o valor da vida é infinito e nenhuma morte por acidente é aceitável, então nenhum nível de risco deve ser tolerável(11).

Outro aspecto relevante, quando se discutem os riscos a partir de uma perspectiva ética, é: muitas atividades beneficiam as gerações presentes, mas os riscos a elas relacionados serão herdados pelas gerações futuras.

Outra ferramenta utilizada na tomada de decisão no gerenciamento de riscos é a chamada análise comparativa de riscos(12). Essa análise consiste na comparação entre diferentes tipos de risco, tipicamente através da elaboração de um lista de riscos para o homem e meio ambiente. Essa lista pode ser formada por avaliações objetivas ou subjetivas. 
Essa relação pode ser utilizada tanto para decidir se um risco é alto ou não, quando comparado aos demais existentes naquele grupo, como para orientar os investimentos para a redução do risco. Essa ferramenta é utilizada para a definição dos critérios de aceitabilidade existentes em vários países, tanto para riscos tecnológicos, como para riscos ambientais. Tais critérios se baseiam na determinação do nível de risco médio para a população e partem do princípio de que uma nova atividade não poderá aumentar esse valor médio(13)(14)(15).

A primeira dificuldade no uso dessa ferramenta é que um risco aceito não necessariamente é um risco aceitável. Além disso, ela desconsidera outros fatores importantes para a aceitabilidade social do risco, como a voluntariedade, o valor moral da atividade, o controle pessoal, distribuição dos riscos e benefícios, além, claro, de os riscos serem percebidos de forma diferente. $O$ fato de se estar exposto a um determinado nível de risco não significa que aquele nível de risco é aceitável, que foi voluntariamente escolhido, que não pode ser reduzido e que a distribuição dos riscos e dos benefícios está adequada.

Mais do que isso, a legitimidade social da atividade e do processo de tomada de decisão é fundamental em uma sociedade democrática e não pode ser apenas deduzida ou inferida a partir de outras atividades existentes, ainda que sejam legítimas.

Se a voluntariedade é fator importante para a aceitabilidade do risco, torna-se necessário para a elaboração de uma política de gerenciamento de riscos caracterizar o que seja risco voluntário e risco involuntário.

Segundo Ferreira(8), voluntário é o "que age espontaneamente; derivado da vontade própria; em que não há coação". Dessa forma, os riscos decorrentes de atividades livremente escolhidas pelas pessoas são considerados riscos voluntários e aqueles aos quais as pessoas não estão submetidas por escolha particular são chamados riscos involuntários.

Os riscos decorrentes de práticas esportivas e/ou associados ao estilo de vida de cada pessoa são considerados voluntários. Os riscos tecnológicos e ambientais são considerados involuntários. Uma diferença importante entre eles é que os riscos voluntários, em geral, são individuais, enquanto os involuntários são sociais. Mas, a separação entre voluntário e involuntário não pode ser objetivamente definida.

Outro aspecto importante é que um risco involuntário pode ser transformado em voluntário por meio da informação ou do conhecimento. Em outras palavras, então um risco é involuntário no caso de ignorância do indivíduo exposto a ele(16).

Mas será que o grau de conhecimento apenas é suficiente para definir a voluntariedade do risco. A população da periferia da Grande São Paulo, 
por exemplo, está exposta a níveis mais altos de riscos do que a das regiões centrais, e certamente conhece bem os perigos que a cercam. A escoIha desse grupo da população em residir nessa região certamente não pode ser considerada voluntária.

Ter qualidade de vida implica alimentação balanceada, boas condições de habitação, educação de qualidade, lazer, o que requer recursos financeiros elevados, não acessíveis à grande maioria da população. Ou seja, o fato de haver nos supermercados uma grande quantidade e variedade de alimentos não garante a liberdade de escolha para os indivíduos.

Portanto, os riscos associados ao estilo de vida podem ser interpretados como involuntários, quando os indivíduos envolvidos não têm efetivamente poder de escolha.

Da mesma forma que é difícil garantir que os riscos associados ao estilo de vida são sempre voluntários, é difícil afirmar que os riscos ambientais são realmente sempre involuntários.

Se a tomada de decisão, em relação à atividade geradora do risco ambiental, foi fundamentada em um balanço risco-benefício, os seus riscos vêm acompanhados de vantagens, as quais foram julgadas capazes de compensar os riscos assumidos. Essas compensações não podem ser também consideradas involuntárias ou desconhecidas.

Starr(17) argumenta que "riscos involuntários são aqueles impostos pela sociedade na qual o indivíduo vive" e que "as atividades são involuntárias quando os critérios e opções são determinados não pelos indivíduos afetados, mas por um corpo controlador, como governo, entidades políticas, grupos de liderança". O autor introduz nesse caso outra dimensão à voluntariedade, que é o controle.

De acordo com Starr, se o controle sobre os fatores causadores do risco é do indivíduo, então os riscos são voluntários. Os riscos tecnológicos e ambientais são involuntários, pois o controle é de responsabilidade de órgãos externos, no caso agências governamentais.

Aqui também as dificuldades não são simples, pois deve-se definir, inicialmente, quais são todos os possíveis agentes controladores externos. Se não é apenas o governo, quais as demais entidades políticas e grupos de liderança que exercem esse controle sobre os níveis de risco dos indivíduos e quais são as responsabilidades de cada um? Mais ainda, quais aspectos da vida dos indivíduos são realmente passíveis de controle externo?

Além disso, como compatibilizar liberdades individuais com o bemestar social? Qual é o limite entre o individual e o social, entre o privado e o público, em uma sociedade que pretende ser efetivamente democrática, justa socialmente e sem problemas ambientais? 
É possível também que um indivíduo queira assumir um nível de risco mais alto, a partir de uma escolha efetivamente voluntária e o seu direito, nesse caso, deve ser respeitado, como, por exemplo, um fumante voluntário. Mas, o sistema público de saúde gasta cerca de cinco vezes mais com os indivíduos fumantes do que com os não fumantes. Nesse caso, como todos contribuem igualmente, 0 indivíduo fumante está recebendo um benefício muito maior do que os não fumantes, o que não é, evidente, socialmente justo. Seria, então, mais justo que o indivíduo fumante pagasse uma contribuição maior, ou todos são iguais perante a lei e devem, assim, contribuir da mesma forma?

Mas a diminuição dos niveis de risco e a melhoria da qualidade de vida da população dependem também de recursos, os quais são limitados e, portanto, a sua aplicação deve ser otimizada. Isso implica investir mais onde a redução do risco é maior por unidade investida, o que resulta numa aplicação desigual dos recursos, uma vez que parte da população estará recebendo uma parcela maior dos investimentos. Assim, uma distribuição eqüitativa dos riscos implica a distribuição desigual dos recursos(18).

Muitas vezes, são gastos milhões de reais para salvar algumas pessoas. Se investidos em outras áreas, poderiam salvar milhares. Quais critérios devem orientar a distribuição desses recursos?

A distribuição dos mesmos níveis de riscos para toda a população seria uma solução socialmente mais justa e ambientalmente mais adequada? Qual a relação entre justiça e eqüidade social? Qual a relação entre eqüidade social e qualidade ambiental?

Um dos requisitos básicos para uma política social de riscos, como para qualquer outra política pública, é não violar os direitos de cada cidadão e incorrer em discriminação e, ao mesmo tempo, fundamentar-se em critérios suficientemente claros e precisos, que não permitam várias interpretações distintas e não resultem em situações socialmente injustas e ambientalmente inadequadas.

Como estabelecer critérios fixos se as linhas divisórias dos seus conceitos fundamentais são flexíveis? Encontrar o ponto de equilíbrio entre todas essas questões certamente não é tarefa fácil e é um grande desafio para a elaboração de políticas de gerenciamento social de riscos, em uma sociedade com justiça social e com pessoas e meio ambiente saudáveis. Os critérios fundamentais dessas políticas, certamente, não podem ser definidos a partir de prerrogativas meramente científicas; devem incluir uma abordagem ética e procedimentos democráticos, uma vez que os processos de tomada de decisão se caracterizam por julgamentos de valores.

\subsection{Políticas Sociais de Riscos}

A primeira consideração importante para a elaboração de uma política de riscos é acerca da incerteza do próprio conceito de risco. $\mathrm{Na}$ prática, 
todos os eventos podem ou não acontecer, o que equivale dizer que, por melhores que sejam os critérios, nenhum garantirá segurança absoluta.

Além disso, as avaliações e decisões são fortemente dependentes de dados e informações, os quais são continuamente gerados e devem, assim, ser também continuamente coletados e inseridos no processo. Essas novas informações podem não apenas confirmar as já existentes, como também alterá-las significativamente e, em alguns casos, até mesmo contradizê-las.

Assim, uma política de gerenciamento deve ser suficientemente flexivel e dinâmica para permitir essa atualização constante das informações, e incluir instrumentos para lidar com as situações nas quais os novos conhecimentos mostrem que os procedimentos anteriores são inadequados.

Um exemplo comum desses problemas é a atualização dos limites de exposição para produtos químicos, seja para a população em geral ou para os trabalhadores em particular. Com o avanço dos estudos toxicológicos, os limites até um dado momento considerados aceitáveis são reduzidos ou é descoberto que certos produtos são carcinogênicos, como já aconteceu com muitos produtos químicos. Quais os direitos sociais e legais das pessoas que estiveram expostas a niveis acima dos aceitáveis?

Essa incerteza no conhecimento científico não é exatamente uma limitação, mas é da própria natureza da ciência. Segundo Morin(5), "a busca do conhecimento não apenas comporta riscos, como também alimenta-se deles". Dessa forma, quem se responsabiliza pelos erros dos cientistas?

Essa questão requer também a discussão do próprio papel do conhecimento científico na construção dos valores e na organização da sociedade atual. As políticas e decisões sociais necessitam de legitimidade social, mesmo que cientificamente corretas.

A grande vantagem de se encontrar uma fórmula científica e conhecer todas as variáveis que influenciam um determinado fenômeno, e como elas estão relacionadas, é a possibilidade de fazer previsões para outros fenômenos similares e de produzir modificações e alcançar outros resultados mais desejáveis.

Do ponto de vista do gerenciamento social de riscos, qual a vantagem de se calcular a priori a aceitabilidade social dos riscos? Ainda que seja possivel saber com antecedência se a opinião de uma comunidade é favorável ou não a uma atividade, é aceitável que esta seja implantada sem maiores esclarecimentos para a população?

Essa previsão da aceitabilidade social dos riscos combinaria com o direito de participação dos cidadãos nas decisões que afetam as suas vidas, ou simplesmente serviria para legitimar as decisões de um grupo 
dominante que detém o conhecimento ou a linguagem técnica em questão, sem o desconforto gerado pelos conflitos de opinião?

Além disso, convém considerar até que ponto, efetivamente, a diminuição dos conflitos entre especialistas e a população indica diminuição dos riscos e melhoria no gerenciamento dos problemas sociais e ambientais?

Segundo Moraes(19), "o conhecimento científico não deve ser normatizador da vida dos indivíduos, mas uma fonte de informações e esclarecimentos para o debate dos vários temas por parte dos cidadãos".

\subsection{Os Atores Sociais Envolvidos}

A Declaração do Rio de Janeiro, da Conferência das Nações Unidas para o Meio Ambiente e o Desenvolvimento, de 1992, em seu artigo 10 diz: "o melhor modo de tratar as questões de meio ambiente é assegurando a participação de todos os cidadãos interessados, no nível pertinente". No Brasil, a própria Constituição Federal de 1988 institucionalizou o processo participativo.

Nas questões de risco não é diferente. A aceitabilidade do próprio processo é parte integral da aceitabilidade dos resultados. Assim, a integração do público na análise de riscos é uma prioridade no sentido de garantir a aceitabilidade. A dificuldade é como considerar essas preocupações de uma maneira eficiente do ponto de vista da proteção da saúde pública e do meio ambiente e socialmente justa e igualitária.

Além disso, a dependência do funcionamento da sociedade atual em relação a uma ciência e tecnologia avançadas demanda organizações mais efetivas e confiáveis, como também suficientemente dinâmicas, para o gerenciamento de riscos(20). A confiança e a credibilidade das instituições gerenciadoras é determinante para a aceitabilidade social dos riscos.

Por outro lado, segundo Ferreira(21), certamente não basta ampliar a participação popular, é necessário ter regras claras, garantindo uma participação igualitária do poder de decisões e, também, os recursos necessários para viabilizar uma efetiva expressão de todos os pontos de vista. A definição dessas regras requer uma revisão e redefinição do papel do Estado e de suas instituições.

Além disso, é de grande relevância ressaltar que, para que haja efetivamente uma decisão conjunta de todos os atores sociais no processo de tomada de decisão, se faz necessária também a democratização do conhecimento, que não pode ser confundido com disseminação de informações pelos meios de comunicação de massa, pois trata-se de um processo 
mais amplo de educação, que forneça elementos para ampliar o debate social e garantir a participação de todos $(22,23)$.

Todavia, não se pode perder de vista também que um bom gerenciamento dos riscos necessita de ambos procedimentos democráticos e ferramentas de negociação éticas e transparentes, bem como de um conhecimento técnico e científico competente.

\section{CONCLUSÃO}

A eficiência do conceito de risco como critério fundamental para a tomada de decisões sociais dependerá não apenas da consideração dos aspectos técnico-científicos, mas também da abordagem ética e procedimentos democráticos. $O$ processo de tomada de decisões para o gerenciamento de riscos é essencialmente um processo de julgamento de valores, que necessita, além de conhecimento científico qualificado, de legitimidade social.

O gerenciamento social de riscos deve ser considerado não apenas como o conjunto de regras e procedimentos que deve ser seguido após a avaliação, com o objetivo de controlar os riscos estimados, mas também como todas as atividades técnicas e legais, bem como o conjunto de todas as decisões e escolhas sociais, políticas e culturais que se relacionam direta ou indiretamente com as questões de risco na nossa sociedade.

No entanto, a complexidade dessas questões não tem sido considerada no atual sistema de gerenciamento de riscos no Brasil. Critérios e ferramentas utilizadas em países desenvolvidos são adotados sem uma análise da sua adequação à realidade brasileira e sem uma avaliação das suas implicações sociais, políticas e éticas. Por esta razão, é necessário que este debate seja ampliado, no meio acadêmico e social, a fim de que decisões não apenas ambientalmente mais adequadas, mas socialmente mais aceitáveis possam ser viabilizadas.

\section{BIBLIOGRAFIA}

(1) BECK, U. Risk society. London: SAGE Publications, 1992.

(2) SJÖBERG, L. Perceived risk vs. demand for risk reduction. Stockholm: Center for Risk Research; 1994. (RHIZIKON Report, 18).

(3) NARDOCCI, A. C. Risco como Instrumento de Gestão Ambiental. São Paulo. Tese de Doutorado - Faculdade de Saúde Pública da USP, 1999.

(4) National Research Council. Understanding risk. Washington (DC): National Academy Press, 1996. 
[5) MORIN, E. O método. Porto Alegre: Sulina, 1998, vol. 4: As idéias.

(6) PORTO M. F. S., FREITAS C. M. "Major chemical accidents in industrializing countries: the socio-political amplification of risk". Risk Anal ,1996; 16:19-29.

(7) WYNNE, B. "Risk and social learning: reification to engagement". In: KRINSKY S., GOLDING D., editors. Social theories of risk. London: Praeger, 1992, pp. 275-97.

(8) FERREIRA, A. B. H. Dicionário Aurélio eletrônico. [CD-rom] Versão 2.0. Rio de Janeiro: Nova Fronteira, 1996.

(9) SWANEY, J. A. "The basic economics of risk analysis". In: MOLAK V, editor. Fundamentals of risk analysis and risk management. Cincinnati: Lewis Publishers, 1996, pp. 99-122.

(10) RENN, O. "The social arena concept of risks debates". In: KRINSKY, S., GOLDING, D., editors. Social Theories of risk. London: PRAEGER, 1992, p. 179-96.

(11) SHRADER-FRECHETTE, K. "Risk and ethics". In: International Conference on Radiation and Society: Comprehending Radiation Risk (Proceedings Series), 1994 Oct., Paris (France). Paris: IAEA, 1994, pp. 167-82.

(12) WILSON, R., CROUCH E. A. "Risk assessment and comparisons: an introduction". Science, 1987, 236:267-70.

(13) National Research Council. Risk assessment in the federal government: managing the process. Washington (DC): National Academy Press, 1983.

(14) Dutch National Environmental Policy Plan. Premises for risk management. The Hague (Netherlands): Ministry of Housing, 1988/9. (Second Chamber of the States General, session 1988-1989, 21 137, n. 5).

(15) Major Industrial Accidentes Council Of Canada. Hazardous substances risk assessment: a mini-guide for municipalities and industry. Ottawa, 1994.

(16) DOUGLAS M., WILDAVISKY A. Risk and culture. Berkeley: University of California Press, 1982.

(17) STARR, C. "Social benefit versus technological risk: what is our society willing to pay for safety?" Science, 1969, 165:1232-38.

(18) DODERLEIN, J. M. "Risk and decisions: introduction". In: SINGLETON WT, HOVDEN, J. Risk and decisions. Chichester: John Wiley \& Sons, 1987, pp. 1-8.

(19) MORAES, A. C. R. Meio ambiente e ciências humanas. São Paulo: Hucitec, 1994. 
(20) SJÖBER, G. L. Perceived risk vs. demand for risk reduction. Stockholm: Center for Risk Research, 1994 (RHIZIKON Report, 18).

(21) FERREIRA, L. C. A questão ambiental: sustentabilidade e políticas públicas no Brasil. São Paulo: Boitempo Editorial, 1998.

(22) GADOMSKA, M. "Risk communication". In: International Atomic Energy Agency. International Conference on Radiation and Society: Comprehending Radiation Risk. Paris: IAEA, 1994, pp. 147-66.

(23) WIEDEMANN, P. M.; FEMERS, S. "Public participation in waste management decision making: analysis and management of conflicts". $J$ Hazard Mat. 1993, 33: 355-68. 